\title{
A New Qualification for the New Year: ISBER and American Society of Clinical Pathology Board of Certification Announce New Qualification in Biorepository Science Examination for Biobank Technicians
}

\author{
Brent Schacter, Nicole Sieffert, ${ }^{2}$ Kristina Hill, ${ }^{3}$ Pat Tanabe, ${ }^{4}$ and Daniel Simeon-Dubach ${ }^{5}$
}

$\mathrm{M}$ ODERN BIOBANKING IS a complex activity that requires highly trained and skilled repository staff to provide a level of quality that will ensure valid and reliable access to high-quality research specimens. Well-trained repository staff are essential to ensure high-quality biospecimens that will be useful in defining the goals of precision medicine, biomarker development, and biomedical research as a whole. Until recently, no formal repository qualification program existed to define the level of skill required to ensure quality in technical biobanking skills, although some training programs for biobanking had been created, including but not limited to CTRNet, Lund University, Integrated Biobank of Luxembourg (IBBL), Medical University Graz, University Côte D'Azur, Nice.

For this reason, International Society for Biological and Environmental Repositories (ISBER) and the American Society for Clinical Pathology Board of Certification (ASCP BOC) have joined forces to create a qualification examination for biobankers. ASCP BOC is an organization that provides excellence in global medical laboratory professional certification. After meeting specific educational and experience requirements for the qualification, candidates will be eligible to complete an online examination and, if successful, gain recognition for their skills and competencies as biobankers. The qualification will be designated as Qualification in Biorepository Science (QBRS) and will be signified by the honorific QBRS.

To develop this qualification, an Memorandum of Understanding (MOU) between ISBER and ASCP BOC was signed in the fall of 2017. Subsequently, a QBRS Workgroup, designated the Examination Committee, was established as a standing committee of the ASCP BOC. This committee has a complement of eight members chosen after ISBER's call for volunteers received a very large response. Committee members were selected taking into consideration diversity in work background in biobanking, geography, experience as a practicing biobanker or an individual with expertise in the principles and practice of biobanking as documented by prior contribution to scholarship or leadership in biobanking. The initial committee members, chosen for a 3-year term, were mutually agreed upon by ISBER and ASCP BOC, nominated for formal appointment, and approved by the Board of Governors of the ASCP BOC.* To provide broad oversight of this activity, a steering committee of ISBER members with appropriate backgrounds in standards development was appointed as a subgroup of the ISBER Standards Committee. ${ }^{\dagger}$ It also has representation by ASCP BOC in the person of Pat Tanabe, the executive director of ASCP BOC. The chair of the steering committee is Brent Schacter.

The first meeting of the Examination Committee was held in December 2018. In the intervening period, the parameters for the examination were set, supporting documents were developed and a bank of examination questions was created and standardized. The steering committee has provided broad oversight of this process, which has been thorough and comprehensive. The Examination Committee has had the responsibility for developing, reviewing, and updating the biobanking item/exam question bank, performing job task analyses, and creating and updating the examination content guidelines, eligibility requirements, and the

*The members of the Examination (Workgroup) Committee are Anne Braggia, Mary Ann Clements, Carol Elliott, Annemieke De Wilde, Kathy Mangold, Hector Monforte, Piper Mullins, and Alison Parry Jones.

The members of the Steering Committee are Brent Schacter (chair), Monique Albert, Jane Carpenter, Yehudit Cohen, Annemieke De Wilde, Koh Furuta, Carlo Largiader, Nicole Sieffert, Daniel Simeon-Dubach, Tamsin Tarling, Heidi Wagner, Peter Watson, and Pat Tanabe.

\footnotetext{
${ }^{1}$ CancerCare Manitoba/University of Manitoba, Winnipeg, Canada.

${ }^{2}$ Independent Consultant, Galveston, Texas.

${ }^{3}$ Independent Consultant, Florida.

${ }^{4}$ ASCP Board of Certification, Chicago, Illinois.

${ }^{5}$ medservice, Walchwil, Switzerland.
} 
candidate experience documentation forms that are needed for the QBRS Program. The Steering Committee has reviewed and provided comment on the supporting elements to clarify and focus content. In essence, ISBER members of the Examination Committee have created the examination content, and ASCP BOC has provided their well-tested and utilized processes for setting the qualification in place.

Resource documents for the QBRS examination are now available on the ISBER website at www.isber.org/ qualification and the ASCP BOC website at www.ascp.org/ boc/qbrs. Details can be found on these sites regarding the routes of and requirements for eligibility to apply for and take the QBRS examination. Other documents and information include a Work Experience Documentation Form, a list of Regional Accrediting Bodies for North American colleges and universities as well as for colleges and universities outside of North America, an examination topic outline, and a list of suggested reading for examination preparation (journals, texts, online).

The requirements for examination application may take one of three routes. Route 1 includes a valid ASCP or ASCP technologist/scientist or specialist certification, a baccalaureate degree from a regionally accredited college/university with a major in biology, chemistry, biotechnology, or a related field and 1 year of full-time experience in a biorepository within the past 5 years. Route 2 does not require an ASCP certification, but includes a baccalaureate degree from a regionally accredited college/university with a major in biology, chemistry, biotechnology, or a related field and two full years of full-time work in a biorepository within the past 5 years. An alternative to the major required in both routes is 30 semester hours in biology and chemistry that may be obtained within or in addition to the baccalaureate degree. Route 3 requires a master's degree from a regionally accredited college/university with a major in biology, chemistry, biotechnology, or a related field and 1 year of experience in a biorepository within the past 5 years. A more detailed description of the requirements for application can be found on the ISBER and ASCP BOC websites as noted earlier.

The QBRS examination questions encompass the major content areas within Biorepository Science, including specimen handling (collection, processing, and storage), sample/data inventory management and quality control, safety and infection control, and biorepository operations. Each of these topics will comprise $25 \%-30 \%$ of the examination, with the exception of biorepository operations $(15 \%-20 \%)$, and will equal a specific percentage as noted of the 50 total examination questions. The questions may be theoretical and/or procedural. The previously referenced websites provide full details regarding the topics that are covered.

The QBRS examination will be open for application as of January 2, 2020. The earliest opportunity for qualified applicants to complete the examination will be in April 2020 and the cost is $\$ 240$ US. The 50 question, multiple choice, timed test is self-administered on your own computer on the date and time of your choice within the 60-day examination period indicated on your admission notification. The examination may be attempted up to three times if initially unsuccessful. QBRS certificate holders will be required to revalidate their qualification every 3 years by documentation of required continuing education or other educational activities as defined by the ASCP BOC.

The ISBER/ASCP BOC partnership allows ISBER to fully participate in the development of the global QBRS credential program requirements that are essential for the future of sustainable quality biobanking. The new QBRS is a unique and important enhancement to the global practice of quality biobanking. Biobankers are encouraged to review the QBRS application materials and strongly consider applying for credentialing as a benchmarked quality biorepository scientist. For further questions, please contact standards@isber.org

Address correspondence to:
Brent Schacter, MD, FRCPC
Department of Medical Oncology and Hematology
University of Manitoba
CancerCare Manitoba
Room 5008c
675 McDermot Avenue
Winnipeg
Manitoba R3E OV9
Canada

E-mail: bschacter@cancercare.mb.ca 